2 A/PROF. JENNIFER STONE (Orcid ID : 0000-0001-5077-0124)

3

4

Article type : Medical Imaging - Radiation Oncology Editorial

6

7

8

Should breast cancer screening programs routinely measure mammographic density?

\title{
9 Author:
}

Dr Jennifer Stone $\mathrm{PhD}^{1}$

$11{ }^{1}$ Centre for Genetic Origins of Health and Disease, Curtin University and The University of Western

12 Australia, Perth, Western Australia, Australia

\section{Corresponding author:}

Dr Jennifer Stone

Centre for Genetic Origins of Health and Disease

The University of Western Australia

35 Stirling Highway, M409

Crawley, 6009

Western Australia, Australia

Telephone: +61 (0)3 92240373

Email: Jennifer.stone@uwa.edu.au

\section{Abstract}

Introduction: Whilst population-based mammographic screening provides the best chances of early detection of breast cancer, the current "one-size-fits-all" recommendation for biennial screening of

This is the author manuscript accepted for publication and has undergone full peer review but has not been through the copyediting, typesetting, pagination and proofreading process, which may lead to differences between this version and the Version of Record. Please cite this article as doi: $10.1111 / 1754-9485.12652$

This article is protected by copyright. All rights reserved 
most women aged 50-74 leaves considerable room for improvement. Evidence suggests that a tailored screening program - where women at different levels of risk are recommended different screening intervals or supplemental screening - may be a more efficient and cost-effective way of detecting breast cancer. Measuring mammographic density, the white appearance of parenchymal tissue on a mammogram, at the time of screening could help identify groups of women who could benefit from different screening recommendations. This article highlights opportunities and obstacles for mammographic density to become a practical population health tool to improve mammographic screening practice in Australia.

Methods: This article considers mammographic density and i) its association with breast cancer risk, ii) its impact on the effectiveness of mammograms at detecting cancer, iii) its known determinants, iv) the prevalence of dense breast tissue in screening populations, v) its correlation within women over time, vi) its measurement, vii) its role within breast cancer risk prediction models, viii) what to tell women, and finally, ix) impediments to its implementation within breast screening programs in Australia.

Conclusion: There is a need for a forward looking, standardised, evidence-based approach to improve breast cancer screening. Routine measurement of mammographic density could facilitate a paradigm shift towards tailored breast cancer screening programs in Australia. Standardised protocols for communicating the risks and screening limitations associated with mammographic density to screening participants are needed.

\section{Key words:}

Breast cancer screening, mammographic density, risk prediction, tailored screening, health promotion

\section{Introduction}

The principal goal of breast cancer screening is early detection of disease in asymptomatic women leading to an eventual reduction in breast cancer mortality. Whilst population-based mammographic screening provides the best chances of early detection, the opportunity for an earlier diagnosis is not equal for all women. Women not only differ in terms of their underlying risk, but also the sensitivity of their mammogram to detect abnormalities. The benefit of screening therefore varies widely across the population. The majority of women are at very low absolute risk, yet most women's screening is scheduled in exactly the same way (in Australia, every two years between ages 50 and 74). A tailored screening program may be a more efficient and effective way of detecting breast cancer. In such a program, women would be recommended different screening strategies based on 
their individual risk factors (e.g. changed screening interval or supplemental screening such as MRI or ultrasound).

One risk factor that would be considered in such a tailored program is mammographic density, the white appearance of parenchymal tissue on a mammogram. Mammographic density is one of the strongest predictors of breast cancer risk (1) and significantly reduces the sensitivity of a mammogram (2). Routine measurement of mammographic density at the time of screening has enormous potential to become a practical and widely used population health tool to identify women at increased (or decreased) risk of breast cancer. Whilst there is considerable research ongoing in this area, there are currently no evidence-based screening recommendations for women with dense breasts. Despite this, legislation in 32 US states mandates that screened women be told of their mammographic density. This legislation has forced greater attention on mammographic density internationally but it is not agreed whether government-funded breast cancer screening programs in Australia should routinely measure and report mammographic density. This review article summarizes several supporting and opposing arguments for the implementation of routine mammographic density measurement and reporting within Australian breast cancer screening programs.

\section{i.) The association between mammographic density and breast cancer risk}

Odds ratios of 4 to 6 are commonly reported to describe the association between mammographic density and breast cancer risk (1). However, these odds ratios describe associations of the extremities of an expansive mammographic density continuum - comparing women with extremely dense breasts to those of similar age with very fatty breasts (1). Ultimately what is needed is a standardized reliable measure of mammographic density that can be applied at the time of screening to inform optimal screening strategies.

In the meantime, reporting odds ratios that describe more meaningful metrics could help clinicians inform women about the association between mammographic density and breast cancer risk on a population level. More recent studies report odds ratios per standard deviation to avoid inconsistencies due to differing mammographic density category thresholds and reference categories (3).

A recent paper by Hopper has developed a method that enables the comparison of the strength of associations across studies and for different risk factors. It is called OPERA (Odds PER Adjusted standard deviation) (4). As an example application to continuous risk factors, Hopper shows that the odds per adjusted standard deviation (OPERA) for the association between mammographic density 
and breast cancer risk is $\sim 1.4$ and is likely to increase with new measures. This estimate of association is within the range of and potentially stronger than that of known gene mutations (OPERAs of 1.2-1.7).

This study indicates that when predicting which women will get breast cancer on a population basis, mammographic density (adjusted for age and body mass index) is potentially a stronger risk factor for breast cancer risk than all genetic risk factors identified in the last two decades, including carrying a mutation in the BRCA1/BRCA2 genes.

\section{ii.) Mammographic density and its impact of the effectiveness of mammograms at}

\section{detecting cancer}

Interval breast cancers are often defined as cancers diagnosed within 12- or 24-months after a normal mammographic screen. Interval cancers typically have a worse prognosis compared to screen-detected cancers; they are more likely to be invasive, of a higher grade and stage and with a greater proportion of HER2 and triple negative molecular subtypes (5). The rate of interval cancers for women aged 50-69 in Australia is currently 9/10,000 women screened which translates to around 1530 potentially missed cancers each year (6).

Interval cancers are either true interval cancers (not present at the time of screening) or false negatives whereby the cancer was missed at the time of screening. Quite often the latter is due to higher mammographic density which "masks" the presence of the cancer as both tumours and mammographic density appear white on a mammogram. The odds of a woman with extremely dense breasts developing an interval-detected breast cancer diagnosed within 24 months after a normal screen are nearly 5-times higher than that of screen-detected cancers (7) and potentially 17 times higher in interval cancers diagnosed within 12 months of a normal screen (OR=17.8; 95\% $\mathrm{Cl}=4.8-65.9)(8)$.

Kerlikowske and colleagues report an optimal combination of individual 5-year risk estimates with BIRADS mammographic density measures to identify women at high risk of interval cancers.

Through simulation, they projected that the ratio of required women to be recommended ultrasound in order to prevent/detect 1 interval cancer was lowest for a screening strategy where women with 5 -year risk $>1.67 \%$ and extremely dense breasts or 5 -year risk $>2.50 \%$ and heterogeneously dense breasts would be recommended supplemental screening (9). Thus, tailored screening strategies could reduce the number of poorer-prognosis interval cancers missed each year, thereby increasing the likelihood of the principal aim of mammographic screening - early detection of disease in asymptomatic women leading to an eventual reduction in breast cancer 
mortality. The disputed value of mammographic screening will not be discussed here. However, the argument that only a modest fraction of the tumours identified by screening would go on to present clinically could potentially be rejected with tailored screening recommendations.

\section{iii.) Mammographic density, its determinants and their association with breast cancer}

It is important to note that not all women with dense breasts have high interval cancer rates (9) and therefore other important risk factors like age, family history, reproductive history, body mass index (BMI), and potentially known breast cancer-susceptibility genetic variants, in addition to mammographic density also need to be considered when recommending different screening strategies. Kerlikowske and colleagues show that the risk of interval breast cancer in women with high mammographic density (i.e. the risk of "masking") but have no other risk factors is quite low $(\mathrm{OR}=0.72,95 \% \mathrm{Cl}: 0.33-1.37)$ (9). It is therefore imperative that measures of mammographic density are not used in isolation, if to be used as a screening tool to identify women at high (or low) risk. Similarly, it has been suggested that family history should not be used in isolation either as the majority of women with early-onset breast cancer do not have a family history of the disease (10).

Age remains the strongest predictor of breast cancer risk. It is very important for age to be taken into account when relating a woman's mammographic density with respect to her breast cancer risk. The "disturbing opposite trend" (11) that mammographic density decreases with age whilst breast cancer risk increases with age can be explained simply: mammographic density for a woman's age is the true risk factor. BMI should also be taken into account when relating a woman's mammographic density to breast cancer risk, particularly when considering measures of percent mammographic density. BMI is a known breast cancer risk factor, particularly in post-menopausal women (10), and is positively correlated to fatty (non-dense) tissue in the breast (11). Thus, the association between $\mathrm{BMI}$ and mammographic density is highly dependent on how mammographic density is expressed (percent or absolute) $(11,12)$ and measured (area or volume) (13) and often confounds the associations of mammographic density and BMI with breast cancer risk. Previous work has shown that adjusting percent mammographic density for age and BMI provides additional predictive information to the Tyrer-Cuzick breast cancer risk prediction score (14) (The IBIS Breast Cancer Risk Evaluation Tool).

Many of the other well-established "environmental" determinants of mammographic density are also associated with breast cancer risk and in the same direction. For example, like breast cancer, mammographic density is negatively associated with number of live births (12) and positively associated with hormone therapy use $(13,14)$. Mammographic density is also positively associated 
with family history and twin studies have shown that around $60 \%$ of the large variation of mammographic density across women can be explained by genetic factors $(15,16)$. Martin and colleagues showed that mammographic density explains around $14 \%$ of the association between family history of breast cancer and risk of the disease (17). This estimate is consistent with the investigations of the genetic overlap between variants shown to be associated with mammographic density and known breast cancer-susceptibility variants $(18,19)$. Only $9-25 \%$ of the variation in absolute and percent measures of mammographic density (respectively) can be explained by all of the above "environmental" factors combined; the corresponding estimate for the known genetic variants is less than $5 \%$. There is considerable research ongoing in this area and increased understanding of the determinants of mammographic density will help identify and understand pathways that explain the etiology of breast cancer.

\section{iv.) The prevalence of mammographically dense breast tissue}

Women of the same age vary greatly in their breast density (20). Some women have lots of mammographic density for their age, whilst others do not. As mentioned above, the reasons are largely thought to be genetic; studies of twin pairs and other relatives have shown that genetic factors are likely to be a major determinant of a woman's mammographic density $(15,16)$. It is thought that breast density is established when the breasts form, largely due to genetic factors, and then "environmental" factors modify breast density over time (21).

It has been estimated that $43 \%$ of the US female population aged $40-75$ years have heterogenously dense or extremely dense breasts (20). Therefore, any recommendations for alternative screening strategies for women with higher mammographic density for their age have to be feasible and acceptable for hundreds of thousands of women. As mentioned, on a population level, breast density is one of the strongest known risk factors for breast cancer $(1,4)$ and on an individual level, it is important to take into account all breast cancer risk factors, not just mammographic density, when considering screening recommendations and possible prevention strategies for women (9). Mammography continues to be the most effective screening test for early detection in asymptomatic women aged 50-74, including those with heterogenously dense or extremely dense breasts. All supplemental screening modalities will identify additional cancers but the question is at what cost. Supplemental ultrasound (+ mammography) has been shown to significantly improve cancer detection rates, it is relatively inexpensive, but also has a high false positive rate resulting in unnecessary biopsies and increased patient anxiety (22). Supplemental MRI has been shown to significantly improve cancer detection rates (23) but is relatively expensive and not a viable option 
for population-based assessment within the BreastScreen program in Australia. Trials investigating whether tomosynthesis could benefit women with dense breasts are still in the early stages (24) and it is not clear whether any additional breast cancer detection via tomosynthesis relates to added benefit or added overdiagnosis (or both). It is also unclear/too early to determine if tomosynthesis reduces interval cancer detection rates. Further investigations of alternative supplemental modalities such as molecular breast imaging (25) and contrast enhanced spectral mammography (26) could also provide additional options for women with dense breasts.

As dense breast tissue is so common within screening populations, robust evidence of both the cost and benefit of screening recommendations involving supplemental screening are needed.

\section{Mammographic density correlation over time and modifiability}

Mammographic density is highly correlated over time within a woman $(21,27)$. Thus, if a woman's breasts are dense for her age at 50 then it is likely that, her breasts will be dense for her age at 65 . This has important implications for screening as high-risk women could potentially be identified by a single early screen upon entry into the screening program (27).

Mammographic density is also modifiable; in the IBIS-I study, a clinical trial of tamoxifen for breast cancer prevention, women whose initial mammographic density was greater than $10 \%$ found an average reduction in mammographic density, in addition to that attributable to aging, of about $8 \%$ after approximately 5 years of tamoxifen use (28). The same study went on to show that women in the tamoxifen arm who experienced a reduction in mammographic density of at least $10 \%$ had a $63 \%$ reduction in breast cancer risk (29). Thus, mammographic density has potential to be used to develop both primary and secondary prevention strategies (e.g. using tamoxifen to reduce mammographic density in high-risk women and/or using mammographic density as a clinical biomarker to indicate whether tamoxifen is beneficial to women with or without breast cancer). The Australian Pharmaceutical Benefits Scheme (PBS), a list of medicines subsidised by the Australian Government, has recently been broadened to allow tamoxifen prescription to include women who are at a moderate to high risk of getting breast cancer. It has been estimated that nearly $40 \%$ of premenopausal and over $25 \%$ of postmenopausal breast cancers could be averted if all women with heterogeneously or extremely dense breasts reduced their breast density to scattered density (30).

The measurement of mammographic density historically has been plagued with several sources of measurement error (e.g. radiographer inconsistencies at the time of mammogram, mammography type (digital vs film) and manufacturer, subjectivity of the measurement). The measurement of 
mammographic density also "does not express an individual anatomic and physiological level of risk"

219 (11). Whilst increased understanding of the biology of mammographic density could provide valuable insight into the etiology of breast cancer, there is clearly existing information in a mammogram that can help predict whether a woman is at increased risk of developing breast cancer (and/or the risk of masking) that is not dependent on the biology of the breast tissue. This is evident through the overwhelming body of evidence that confirms the significant association between mammographic density and breast cancer risk regardless of method of measurement, mammography type, comparison categories, study design, and population $(1,8)$. The measurement of mammographic density has proven to be a very robust risk factor for breast cancer on a population level.

There are now commercially available, automated, reliable measures that strongly predict breast cancer risk (in case-control studies). The largest and most comprehensive investigation of the association between breast cancer risk and different measures of mammographic density thus far showed that fully-automated methods are valid alternatives to the labour-intensive "gold standard" thresholding techniques for quantifying mammographic density from full field digital mammography (FFDM). The authors concluded that the choice of a particular method will depend on the aims and setting of the screening facility (3).

We are currently organizing an international Measurement Challenge whereby thousands of casecontrol sets of mammographic images from Australia, Malaysia, Norway, the UK, and the US have been combined and then split into a "training set" where the breast cancer status is known and the "test set" where the Challengers are blind to breast cancer status. Challengers submit their "test" measurements for a centralized statistical analysis which is currently underway. The goal is to determine the strongest predictor of breast cancer risk. Measurement techniques include not only volume and area based measures of percent and absolute mammographic density, but other measurements of image features such as texture and skewness as well. Recent reports also suggests that areas of extremely high density rather than total dense area are stronger predictors of risk (31, 32). This study will provide additional evidence to support the effectiveness and reliability of available automated measurement techniques.

It is still not agreed how best to use information collected at the time of screening, including mammographic density, to maximize early detection and efficiency of screening programs. There are several breast cancer risk prediction models designed to predict which women will develop the 
disease in future (33). Most of them are genetic risk prediction models and designed for effective assessment of women at high risk with strong family histories. As far as the author knows, only two

252

253

254

255

256

257

258

259

260

261

262

263

264

265

266

267

268

269

270

271

272

273

274

275

276

277

278

279 risk models have been used for assessing risk within healthy populations: the Breast Cancer Risk Assessment Tool (commonly referred to as the Gail Model) and the IBIS Breast Cancer Risk Evaluation Tool (commonly referred to as the Tyrer-Cuzick Model). Both of these risk models have been compared prospectively in a small study of 1933 women enrolled in a family history clinic; it was concluded that the IBIS Breast Cancer Risk Evaluation Tool was the most consistently accurate model for predicting the risk of breast cancer $(33,34)$. The tool is universally accessible and population screening-friendly (10); it includes questions regarding family history, weight and height, reproductive factors, history of related disease, and a new version is about to be released that now includes mammographic density. The UK study, PROCAS, has also already shown that it is feasible to collect the required risk factor data from women when they attend their screening appointment (35). In terms of compliance, this study also found that the majority of women wished to receive information about their breast cancer risk and participation in future screening was high (10).

There is also vast potential to, in future, include common genetic variants associated with breast cancer risk into prediction models. There are currently approximately $110+$ such variants with dozens more to be reported soon $(36,37)$ as part of the international Breast Cancer Association Consortium OncoArray project. Combined, these loci explain $\sim 18 \%$ of the familial relative risk of breast cancer. There is a lot of work being done on creating a polygenic risk score that incorporates all of the common variants to assess individual risk. This information can also be used by BOADICEA (Breast and Ovarian Analysis of Disease Incidence and Carrier Estimation Algorithm), a risk prediction model used to compute BRCA1 and BRCA2 mutation carrier probabilities and age-specific risks of breast and ovarian cancer. BOADICEA has been validated in a large series of families from UK genetics clinics (38) for predicting BRCA1/2 mutation carrier status and in Australian women for predicting future breast cancer risk (33). In the UK, it is recommended as a risk assessment tool in the National Institute for Health and Care Excellence clinical guideline CG164 (34) and has been incorporated in the guidelines of several countries for the management of familial breast cancer. It has approximately 5000 registered users worldwide and it is commonly used in high-risk clinics in Australia.

Researchers in Melbourne have developed a tailored, web-based, decision support tool for breast cancer risk assessment called iPrevent ${ }^{\circledR}$ that uses either the IBIS Breast Cancer Risk Evaluation Tool or BOADICEA depending on the information provided by the user (38). A similar tool or an adapted version of this tool could facilitate individual risk assessment as part of a population based screening 
program. Individual risk estimates in combination with mammographic density measurements could be used to recommend tailored screening strategies to women. For example, women at high risk with heterogeneous or extremely dense breasts may benefit from supplemental screening (such as ultrasound or MRI) (9) or women at high risk with no or scattered density may benefit from annual screening. Conversely, women at low risk with no density may not need to be screened as often (39). However, there are currently no evidence-based screening recommendations for women at different levels of risk with different amounts of mammographic density. A randomized control trial (RCT) nested within an Australian breast screening program would enable a comparison of the interval cancer rate in tailored screening interventions to current practice. The feasibility and acceptability of such a trial needs to be assessed.

viii.) What to tell women about mammographic density

294

Whilst researchers have been investigating the associations between mammographic density and breast cancer for many years, the concept of mammographic density or "breast density" is still relatively new to lay populations. The literature regarding what information women find useful and meaningful in order to make good decisions regarding their mammographic density is very sparse and mostly sourced from the United States. A recent review of the literature on mammographic density knowledge and breast density awareness concluded that "more quality studies are needed that focus on how well women understand the relationship between breast density, breast cancer risk, and breast cancer screening, especially in diverse populations." (40)

In Australia, only one of the publicly funded state-run BreastScreen programs, BreastScreen Western Australia, currently notifies participants with extremely dense breasts, informing them that dense breast tissue is normal but it is more difficult to see the early signs of breast cancer in dense breasts. It recommends contacting their General Practitioner (GP) for a breast examination.

American consumer advocacy groups promote that women need to be informed of their mammographic density, the limitations of mammography to find tumours in dense breasts and the increased risk factor of mammographic density, to ensure that women with dense breasts have access to an early diagnosis (41). This message is gaining momentum in Australia $(42,43)$.

In the absence of a standardized protocol for communicating the risks and screening limitations associated with mammographic density to screening participants, women are encouraged to be aware of their breast health, maintain a regular breast screening program if aged 50 to 74 , and consult a GP if they notice any changes or irregularities with their breasts. Further investigation into 
the awareness and knowledge of mammographic density in the general Australian population is needed, as well as an assessment of the impact of informing women that they have dense breasts.

\section{ix.) Impediments to implementation of routine measurement of mammographic density} within breast screening programs in Australia

From (i.) above, mammographic density is likely the strongest and most common breast cancer risk factor but it is still not agreed how best to measure it. The infrastructure for routine manual BIRADS measurement in the United States has been well established, long before the recent introduction of mandatory reporting legislation. However BIRADS measurement is subjective and therefore prone to measurement error, both within observers (repeatability) and between observers (reproducibility) (44). In Australia, infrastructure to record mammographic density is not common and any means to integrate measurement of mammographic density into screening practice needs to be practical, reliable and affordable. There is very little discussion in the literature about the practical aspects of implementing automated mammographic density-measuring software within existing screening programs. Most of the automated methods require assessment of raw ("for processing") FFDM images. In Australia, raw images are not typically stored in addition to the processed ("for presentation") images, potentially requiring double the bandwidth and data storage. Automated methods have been shown to provide reliable measures (45) but the practicality and the affordability depend on aim and setting of the screening program.

As discussed in (ii), the aim of targeting the reduction of poorer-prognosis interval cancer via tailored screening strategies is achievable but significant groundwork needs to be completed first. Even if screening programs routinely measured mammographic density, it is still not clear how best to use the information. Automated measures are generally on a continuous scale and meaningful cutpoints that define increasing levels of risk are not yet derived. A standardized measure of mammographic density that adjusts for age, $\mathrm{BMI}$ and other risk factors is desperately needed to determine where an individual woman "sits" on the breast cancer risk spectrum. As discussed in (iii), continued improvements in risk prediction models will provide useful tools that can be used quickly and easily at the time of mammography. These tools not only have the potential to identify and target women at increased risk, but also identify women at low risk who potentially don't need to be screened as often, thereby offsetting the costs of supplemental screening or increased interval screening in higher risk groups. However, compliance of whether women will adhere to screening strategies that involve more or less screening also needs to be assessed. The success of tailored screening programs depends largely on understanding and informing women to ensure compliance of the recommended screening strategy. 
347 A randomized controlled trial (RCT) nested within a breast screening program will be needed to compare tailored screening interventions to current practice. The feasibility of such a trial needs to be assessed and large sources of funding would need to be obtained. A population-wide one-sizefits-all screening message is easier to administer and for the public to understand (and comply) however, the majority of women are at very low absolute risk of developing breast cancer and receive very small benefit from biennial screening. Also, the increasing trend of supplemental screening in women with dense breasts in the US - particularly with disregard for other risk factors could potentially do more harm than good with respect to increased anxiety associated with false positives and increased financial burden. In Australia, the "first do no harm" principle appears to support maintaining the status quo however this may change with increased consumer advocacy regarding mammographic density reporting.

Further scope to improve breast cancer screening was discussed in (v). Prevention is not typically an aim for screening programs and the use of tamoxifen is not without risk, particularly the risk of endometrial cancer and venous thromboembolism events. However, hopefully there are similar pathways and less invasive interventions that can be identified to inform prevention strategies. In the interim there is also scope for mammographic density to be used as a biomarker to determine if prevention interventions are working. With a targeted screening age of 50+ years, prevention strategies would arguably be too late however the merits of a "baseline" early screen at age 40 warrants further investigation.

The merits of collecting blood for a genetic test upon entry to the screening program should also be discussed. As discussed in (vii), there are large numbers of common genetic variants associated with breast cancer risk, each contributing very small effects but in combination and measured within a population based screening program, could significantly improve the identification of women at higher risk of disease. Blood would likely only have to be collected once but the costs of collection, processing, storage, and re-processing as new information becomes available, would be significant. Finally, discussions regarding whether or not to tell women if they have dense breasts continues to catch 22. The first stumble starts with the simple definition of "dense breasts". Mammographic density is not a dichotomous trait and without a standardized and reliable measurement it is difficult to relay to women the risks and screening limitations without causing alarm. Almost all women have some mammographic density and the majority of which will not develop breast cancer. The next hurdle is what to recommend women to do next. In the absence of evidence-based screening recommendations for women with dense breasts, maintaining the status quo appears to be the current stance for most breast screening programs in Australia. 
There is a need for a forward looking, standardised, evidence-based approach to improve breast cancer screening. Standardised protocols for communicating the risks and screening limitations associated with mammographic density to screening participants are needed. Systematic collection of mammographic density measurements and other important risk factors at the time of mammography could facilitate a paradigm shift towards tailored breast cancer screening programs in Australia.

Acknowledgements

JS is a National Breast Cancer Foundation Research Fellow

\section{Conflict of Interest}

No conflict of interest to declare.

References

1. McCormack VA, dos Santos Silva I. Breast density and parenchymal patterns as markers of breast cancer risk: a meta-analysis. Cancer Epidemiol Biomarkers Prev. 2006;15(6):1159-69. mammography, physical examination, and breast US and evaluation of factors that influence them: an analysis of 27,825 patient evaluations. Radiology. 2002;225(1):165-75. 3. Eng A, Gallant Z, Shepherd J, McCormack V, Li J, Dowsett M, et al. Digital mammographic density and breast cancer risk: a case inverted question markcontrol study of six alternative density assessment methods. Breast Cancer Res. 2014;16(5):439.

4004 4. Hopper JL. Odds per adjusted standard deviation: comparing strengths of associations for 401 risk factors measured on different scales and across diseases and populations. Am J Epidemiol. 402 2015;182(10):863-7.

403 5. Meshkat B, Prichard RS, Al-Hilli Z, Bass GA, Quinn C, O'Doherty A, et al. A comparison of clinical-pathological characteristics between symptomatic and interval breast cancer. Breast. 2015;24(3):278-82.

406 6. BreastScreen Australia monitoring report 2011-2012. Canberra: AlHW: Australian Institute 407 of Health and Welfare; 2014. p. 37-40.

408 7. Nickson C, Arzhaeva Y, Aitken Z, Elgindy T, Buckley M, Li M, et al. AutoDensity: an automated 409 method to measure mammographic breast density that predicts breast cancer risk and screening 410 outcomes. Breast Cancer Res. 2013;15(5):R80. 
411 8. Boyd NF, Guo H, Martin L, Sun L, Stone J, Fishell E, et al. Mammographic density and the risk 412 and detection of breast cancer. N Engl J Med. 2007;356(3):227-36.

413 9. Kerlikowske K, Zhu W, Tosteson AN, Sprague BL, Tice JA, Lehman CD, et al. Identifying 414 women with dense breasts at high risk for interval cancer: a cohort study. Annals of internal 415 medicine. 2015;162(10):673-81.

416 10. Evans DG, Howell A. Can the breast screening appointment be used to provide risk 417 assessment and prevention advice? Breast Cancer Res. 2015;17:84.

418 11. Colin C, Schott AM, Valette PJ. Mammographic density is not a worthwhile examination to 419 distinguish high cancer risk women in screening. European radiology. 2014;24(10):2412-6.

420 12. Yaghjyan L, Colditz GA, Rosner B, Bertrand KA, Tamimi RM. Reproductive factors related to 421 childbearing and mammographic breast density. Breast Cancer Res Treat. 2016;158(2):351-9.

422 13. Huo CW, Chew GL, Britt KL, Ingman WV, Henderson MA, Hopper JL, et al. Mammographic 423 density-a review on the current understanding of its association with breast cancer. Breast Cancer 424 Res Treat. 2014;144(3):479-502.

425 14. Couto E, Qureshi SA, Hofvind S, Hilsen M, Aase H, Skaane P, et al. Hormone therapy use and 426 mammographic density in postmenopausal Norwegian women. Breast Cancer Res Treat.

$427 \quad 2012 ; 132(1): 297-305$.

428 15. Boyd NF, Dite GS, Stone J, Gunasekara A, English DR, McCredie MR, et al. Heritability of 429 mammographic density, a risk factor for breast cancer. N Engl J Med. 2002;347(12):886-94.

430 16. Stone J, Dite GS, Gunasekara A, English DR, McCredie MR, Giles GG, et al. The heritability of 431 mammographically dense and nondense breast tissue. Cancer Epidemiol Biomarkers Prev. 432 2006;15(4):612-7.

433 17. Martin L, Melnichouk O, Guo H, Chiarelli AM, Hislop TG, Yaffe MJ, et al. Family history, 434 mammographic density, and risk of breast cancer. Cancer Epidemiol Biomarkers Prev. $435 \quad 2010 ; 19(2): 456-63$.

436 18. Stone J, Thompson DJ, Dos Santos Silva I, Scott C, Tamimi RM, Lindstrom S, et al. Novel

437 Associations between Common Breast Cancer Susceptibility Variants and Risk-Predicting

438 Mammographic Density Measures. Cancer Res. 2015;75(12):2457-67.

439 19. Varghese JS, Thompson DJ, Michailidou K, Lindstrom S, Turnbull C, Brown J, et al.

440 Mammographic breast density and breast cancer: evidence of a shared genetic basis. Cancer Res. $441 \quad 2012 ; 21(12): 2167-75$.

442 20. Sprague BL, Gangnon RE, Burt V, Trentham-Dietz A, Hampton JM, Wellman RD, et al.

443 Prevalence of mammographically dense breasts in the United States. J Natl Cancer Inst. $444 \quad 2014 ; 106(10)$.

This article is protected by copyright. All rights reserved 
21. Stone J, Dite G, Giles GG, Cawson J, Hopper J. Inference about causation from elimination of familial confounding: application to longitudinal twin data on mammographic density measures that predict breast cancer risk. Cancer Epidemiol Biomarkers Prev. 2012;21(7):1149-55. 22. Berg WA, Blume JD, Cormack JB, Mendelson EB, Lehrer D, Bohm-Velez M, et al. Combined screening with ultrasound and mammography vs mammography alone in women at elevated risk of breast cancer. JAMA. 2008;299(18):2151-63. 23. Kuhl CK, Schrading S, Strobel K, Schild HH, Hilgers RD, Bieling HB. Abbreviated breast magnetic resonance imaging (MRI): first postcontrast subtracted images and maximum-intensity projection-a novel approach to breast cancer screening with MRI. J Clin Oncol. 2014;32(22):2304-10. 24. Houssami N, Turner RM. Rapid review: Estimates of incremental breast cancer detection from tomosynthesis (3D-mammography) screening in women with dense breasts. Breast. 2016;30:141-5.

457 25. Rhodes DJ, Hruska CB, Conners AL, Tortorelli CL, Maxwell RW, Jones KN, et al. Journal club: molecular breast imaging at reduced radiation dose for supplemental screening in mammographically dense breasts. AJR Am J Roentgenol. 2015;204(2):241-51.

460 26. Lee-Felker SA, Tekchandani L, Thomas M, Gupta E, Andrews-Tang D, Roth A, et al. Newly 461 Diagnosed Breast Cancer: Comparison of Contrast-enhanced Spectral Mammography and Breast MR Imaging in the Evaluation of Extent of Disease. Radiology. 2017:161592. 27. McCormack VA, Perry NM, Vinnicombe SJ, Dos Santos Silva I. Changes and tracking of mammographic density in relation to Pike's model of breast tissue aging: a UK longitudinal study. Int J Cancer. 2010;127(2):452-61. 28. Cuzick J, Warwick J, Pinney E, Warren RM, Duffy SW. Tamoxifen and breast density in women at increased risk of breast cancer. J Natl Cancer Inst. 2004;96(8):621-8. 29. Cuzick J, Warwick J, Pinney E, Duffy SW, Cawthorn S, Howell A, et al. Tamoxifen-induced reduction in mammographic density and breast cancer risk reduction: a nested case-control study. J Natl Cancer Inst. 2011;103(9):744-52.

471 30. Engmann NJ, Golmakani MK, Miglioretti DL, Sprague BL, Kerlikowske K, Breast Cancer

472 Surveillance C. Population-Attributable Risk Proportion of Clinical Risk Factors for Breast Cancer.

473 JAMA Oncol. 2017.

474 31. Nguyen TL, Aung YK, Evans CF, Dite GS, Stone J, Maclnnis RJ, et al. Mammographic density 475 defined by higher than conventional brightness thresholds better predicts breast cancer risk. Int J $476 \quad$ Epidemiol. 2016. 

defined by higher than conventional brightness threshold better predicts breast cancer risk for fullfield digital mammograms. Breast Cancer Res. 2015;17:142. 33. Amir E, Freedman OC, Seruga B, Evans DG. Assessing women at high risk of breast cancer: a review of risk assessment models. J Natl Cancer Inst. 2010;102(10):680-91. 34. Amir E, Evans DG, Shenton A, Lalloo F, Moran A, Boggis C, et al. Evaluation of breast cancer risk assessment packages in the family history evaluation and screening programme. J Med Genet. 2003;40(11):807-14.

35. Evans DG, Warwick J, Astley SM, Stavrinos P, Sahin S, Ingham S, et al. Assessing individual breast cancer risk within the U.K. National Health Service Breast Screening Program: a new paradigm for cancer prevention. Cancer prevention research. 2012;5(7):943-51. 36. Michailidou K, Beesley J, Lindstrom S, Canisius S, Dennis J, Lush MJ, et al. Genome-wide association analysis of more than 120,000 individuals identifies 15 new susceptibility loci for breast cancer. Nat Genet. 2015;47(4):373-80.

491 37. Michailidou K, Hall P, Gonzalez-Neira A, Ghoussaini M, Dennis J, Milne RL, et al. Large-scale 492 genotyping identifies 41 new loci associated with breast cancer risk. Nat Genet. 2013;45(4):353-61, $493 \quad 61 \mathrm{e} 1-2$. 38. Antoniou AC, Hardy R, Walker L, Evans DG, Shenton A, Eeles R, et al. Predicting the likelihood of carrying a BRCA1 or BRCA2 mutation: validation of BOADICEA, BRCAPRO, IBIS, Myriad and the Manchester scoring system using data from UK genetics clinics. J Med Genet. 2008;45(7):425-31. 39. Trentham-Dietz A, Kerlikowske K, Stout NK, Miglioretti DL, Schechter CB, Ergun MA, et al. Tailoring Breast Cancer Screening Intervals by Breast Density and Risk for Women Aged 50 Years or Older: Collaborative Modeling of Screening Outcomes. Annals of internal medicine. 2016. Review of Literature. J Prim Care Community Health. 2016;7(3):207-14.

41. Are you Dense? United States: Nancy Capella; 2016 [www.areyoudense.org].

42. Breast Kept Secret Australia: Kylea Tink \& Associates in conjunction with Volpara Solutions Limited; 2016 [www.breastkeptsecret.com.au]. [www.informd.org.au].

507 44. Yaffe M, Boyd N. Mammographic breast density and cancer risk: the radiological view. 508 Gynecol Endocrinol. 2005;21 Suppl 1:6-11.

509 45. Alonzo-Proulx O, Mawdsley GE, Patrie JT, Yaffe MJ, Harvey JA. Reliability of automated breast density measurements. Radiology. 2015;275(2):366-76. 


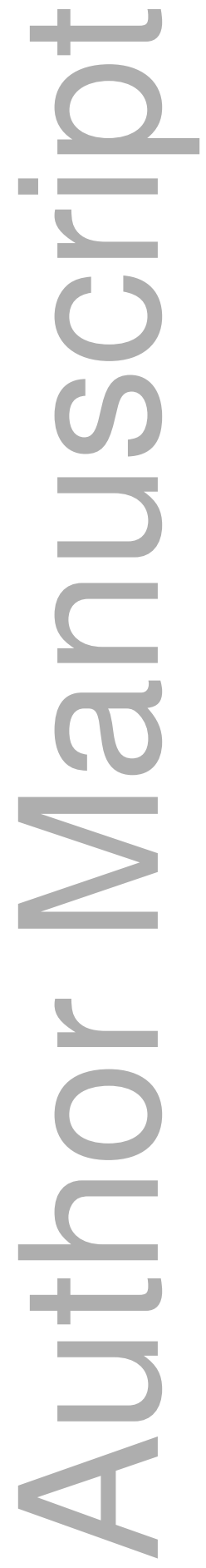

This article is protected by copyright. All rights reserved 


\section{University Library}

\section{- M M N E R VA A gateway to Melbourne's research publications}

Minerva Access is the Institutional Repository of The University of Melbourne

Author/s:

Stone, J

Title:

Should breast cancer screening programs routinely measure mammographic density?

Date:

2018-04-01

Citation:

Stone, J. (2018). Should breast cancer screening programs routinely measure

mammographic density?. JOURNAL OF MEDICAL IMAGING AND RADIATION ONCOLOGY, 62 (2), pp.151-158. https://doi.org/10.1111/1754-9485.12652.

Persistent Link:

http://hdl.handle.net/11343/283824 